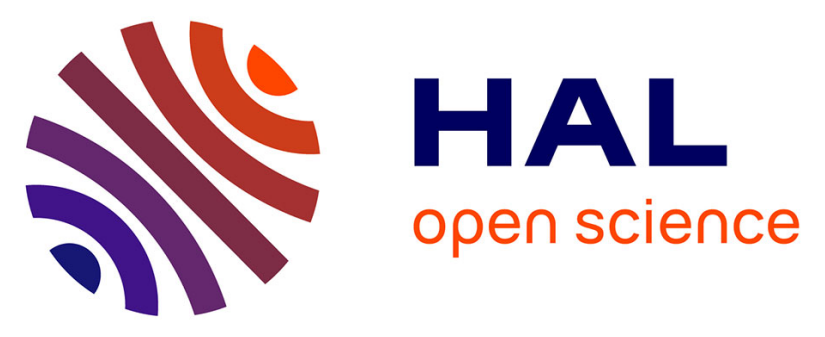

\title{
Induction chemotherapy with carboplatin and taxol followed by radiotherapy and concurrent weekly carboplatin + taxol in locally advanced nasopharyngeal carcinoma
}

\author{
Mario Airoldi, Pietro Gabriele, Anna Maria Gabriele, Massimiliano Garzaro, \\ Luca Raimondo, Fulvia Pedani, Fabio Beatrice, Giancarlo Pecorari, Carlo
}

Giordano

\section{- To cite this version:}

Mario Airoldi, Pietro Gabriele, Anna Maria Gabriele, Massimiliano Garzaro, Luca Raimondo, et al.. Induction chemotherapy with carboplatin and taxol followed by radiotherapy and concurrent weekly carboplatin + taxol in locally advanced nasopharyngeal carcinoma. Cancer Chemotherapy and Pharmacology, 2010, 67 (5), pp.1027-1034. 10.1007/s00280-010-1399-5 . hal-00609754

\section{HAL Id: hal-00609754 https://hal.science/hal-00609754}

Submitted on 20 Jul 2011

HAL is a multi-disciplinary open access archive for the deposit and dissemination of scientific research documents, whether they are published or not. The documents may come from teaching and research institutions in France or abroad, or from public or private research centers.
L'archive ouverte pluridisciplinaire HAL, est destinée au dépôt et à la diffusion de documents scientifiques de niveau recherche, publiés ou non, émanant des établissements d'enseignement et de recherche français ou étrangers, des laboratoires publics ou privés. 


\section{INDUCTION CHEMOTHERAPY WITH CARBOPLATIN AND TAXOL}

\section{FOLLOWED BY RADIOTHERAPY AND CONCURRENT WEEKLY}

\section{CARBOPLATIN + TAXOL IN LOCALLY ADVANCED NASOPHARYNGEAL}

\section{CARCINOMA.}

1- Mario Airoldi, MD, Department of Medical Oncology, San Giovanni Battista Hospital, Turin, Italy

2- Pietro Gabriele, MD , Radiation Therapy Division, Cancer Institute, Candiolo, Turin ,Italy

3- Anna Maria Gabriele, MD, Radiation Therapy Division, San Giovanni Antica Sede Hospital, Turin, Italy

4- Massimiliano Garzaro, MD, I ${ }^{\text {st }}$ ENT Clinic, Physiopathology Department, University of Turin, Italy

5- Luca Raimondo, MD, I ${ }^{\text {st }}$ ENT Clinic, Physiopathology Department, University of Turin, Italy

6- Fulvia Pedani , MD, Department of Medical Oncology, San Giovanni Battista Hospital, Turin, Italy

7- Fabio Beatrice, MD, ENT Department, Giovanni Bosco Hospital, Turin, Italy

8- Giancarlo Pecorari, MD, It ENT Clinic, Physiopathology Department, University of Turin, Italy

9- Carlo Giordano, Professor, I ${ }^{\text {st }}$ ENT Clinic, Physiopathology Department, University of Turin, Italy

Corresponding author:

Massimiliano Garzaro, MD

$1^{\text {st }}$ ENT Division, Department of Clinical Physiopathology, San Giovanni Battista Hospital, Via Genova, 3 - 10126 Turin, Italy

Telephone number: +390116336688 Fax number: +390116336650

Mail: garz@libero.it 


\section{Conflict of Interest Statement}

All Authors disclose any financial and personal relationships with people or organisations that could influence their work. 


\begin{abstract}
Purpose: Aim of this study was the clinical evaluation of carboplatin-taxol combination in a neoadjuvant and concomitant setting with conventional radiotherapy in locoregionally advanced nasopharyngeal carcinoma (A-NPC).

Methods: Thirty patients were treated with three cycles of carboplatin (AUC6) plus taxol $\left(175 \mathrm{mg} / \mathrm{m}^{2}\right)$ on day 1 every 3 weeks, followed by weekly carboplatin (AUC1) plus Taxol (60mg/m2) and concomitant radiotherapy (70Gy).

Results: We observed the objective complete response rates of $33 \%$ (after chemotherapy) and $87 \%$ (after chemo-radiotherapy). Treatment tolerability and toxicity were controllable. Three and five years progression free survival were $80 \%$ and $75 \%$ respectively and 3 and 5 years overall survival were $85 \%$ and $80 \%$ (follow-up 49.5 months). Five years loco-regional control was $90.3 \%$ and five years distant metastases free survival was $85 \%$.
\end{abstract}

Conclusions: Neoadjuvant-chemotherapy with such protocol represents a feasible, efficient treatment for patients with A-NPC, ensuring excellent locoregional disease control and overall survival with low incidence of distant metastases.

Key Words: Nasopharyngeal carcinoma, neoadjuvant chemotherapy, chemoradiation, carboplatin, taxol. 


\section{Introduction}

In nasopharyngeal carcinoma (NPC) the place of chemotherapy (CHT), which is not discussed in metastatic disease, is controversial for the initial management of the disease.

Recent trials and meta-analyses highlight the need to associate chemotherapy with radiotherapy (RT): concomitant chemo-radiotherapy (CRT) appears to be now the standard treatment for locally advanced (T2B and more) and/or node positive $(\mathrm{N}+)$ patients [1].

Despite its more questionable role, the addition of induction CHT remains attractive in locoregionally advanced NPC patients, partly with the purpose of shrinking down the primary tumour before irradiation and partly in order to eradicate micrometastases without delay. However, phase III trials, comparing induction chemotherapy with radiotherapy alone [2-5], failed to show an improvement in overall survival, despite a significant reduction in local and distant failures. Four recent published studies [6-8], performed in extra-European countries, shown that induction CHT followed by CRT obtained high responses in patients coming from both endemic and non endemic areas: in two trials the authors proposed a three-drugs induction scheme, while in the others cisplatin + epirubicin or paclitaxel and carboplatin were respectively administered.

We have published our previous experience with induction cisplatin plus epirubicin followed by conventional radiotherapy plus every 3 week cisplatin administration [9]; our results confirm the high activity of this approach in a non-endemic population.

No phase II trials have investigated a two-drug combination during conventional, non-splitted, radiotherapy after a full course of induction chemotherapy.

When the study was designed the association carboplatin + taxol was known as a feasible and effective combination in advanced-metastatic nasopharyngeal tumours. [10-12] 
These two drugs were known as radiosensitizers and weekly taxol infusion was indicated as an ideal way to give dose-density with enhanced therapeutic index. [13]

In this paper we report our experience with neoadjuvant chemotherapy with carboplatin + taxol followed by radiotherapy with weekly carboplatin - taxol combination in locally advanced NPC, observed in a non-endemic population. 


\section{Methods and Materials}

\section{Eligibility}

In the present multicentric retrospective analysis patients having histological confirmed NPC, stage III-IVB according to $\underline{2010}$ AJCC stage classification ( $\underline{7}^{\text {th }}$ ed.), who had received no previous CHT and/or RT were included. Individuals aged $>18$ presenting measurable disease and WHO performance status (WHO-PS) of 0 or 1 were also considered eligible for our study. $\underline{\text { Clinical }}$ introduction of this two-drug association was approved by the Ethics Committee of each participating centre and all patients provided written informed consent.

Patients were excluded in case of inadequate organ function, as indicated by an absolute neutrophil count $\leq 1.5 \times 10^{9} / \mathrm{L}$, platelet count $\leq 100 \times 10^{9} / \mathrm{L}$, serum creatinine $\geq 1.5$ times the upper limit of normal or 24-hour creatinine clearance $\leq 50 \mathrm{ml} / \mathrm{min}$, serum bilirubin $>1.5$ times the upper limit of normal (ULN), ALT, AST and alkaline phosphatase levels $\geq 2$ times the ULN. Moreover we considered not eligible patients presenting prior malignancy apart from non-melanoma skin cancer or carcinoma in situ of the uterine cervix, pre-existing motor or sensory neurotoxicity $\geq$ WHO grade 2, significant hearing impairment unless due to NPC, active uncontrolled infection, unstable cardiac disease and pregnancy or lactation. Written informed consent was obtained from all patients.

\section{Evaluation and follow-up}

The main contents of pre-treatment evaluation have been the following: physical examination, assessment of PS, urine analysis, complete blood cell count (CBC) with differential, serum electrolytes, liver function tests, magnetic resonance, and measurement of index lesions. Other tests included electrocardiography (ECG) and 24-hour creatinine clearance. Baseline imaging was 
composed by bone scan, contrast-enhanced computed tomography (CT) scan of thorax and abdomen, CT and/or magnetic resonance imaging (MRI) of the nasopharynx and neck. During the treatment period, patients underwent weekly physical examination and toxicity assessment. Laboratory exams and 24-hour creatinine clearance had to be repeated every 3 weeks, while $\mathrm{CBC}$ count had to be performed on days 1 and 8 of each cycle of neoadiuvant chemotherapy and once a week during RT.

Assessment of tumour response by clinical examination and head and neck MRI or CT was performed after neoadjuvant CHT. Once completed CHT and CRT, endoscopy, head and neck MRI or CT, thoracic and abdominal CT scan could be performed.

Six to eight weeks after the completion of CRT, complete physical examination, nasopharyngoscopy, thoracic and abdominal CT scan, MRI of nasopharynx, biochemistry and blood cell count could be performed. Patients had to be followed up by clinical examination every 2 months during the first year, every 3 months for the subsequent 2 years and every 6 months thereafter. Nasopharyngoscopy and imaging (CT, MRI or ultrasound investigation) were disposed for patients developing signs or symptoms suggestive of disease recurrence. Endoscopic or ultrasound guided biopsy had to be performed, if deemed necessary.

Tumour response was assessed according to Response Evaluation Criteria in Solid Tumours (RECIST) criteria. Systemic toxicity from treatment was graded according to WHO and Radiation Therapy Oncology Group (RTOG) criteria.

\section{Induction Chemotherapy}

Neoadjuvant CHT consisted of 3 cycles of paclitaxel $175 \mathrm{mg} / \mathrm{m} 2$ administered over 3 hours followed by carboplatin area under the concentration-time curve 6 (carboplatin dosing to the area under the curve of 6 was calculated using Calvert Formula) administered over 30 to 60 minutes , repeated on day 22. Paclitaxel was preceded by premedication with dexametasone $(20 \mathrm{mg})$, diphenidramine $(25 \mathrm{mg}$ ) and ranitidine $(50 \mathrm{mg})$. If the absolute neutrophil count was less 
$1,200 / \mathrm{mm} 3$ or platelets was less than $100,000 / \mathrm{mm} 3$ on day 22 , treatment was held until counts returned to this level. If the cycle was held more than 3 weeks, patients proceeded to tumor response evaluation and started chemo-radiotherapy. Prophylactic use of recombinant granulocyte colony stimulating factor was not allowed.

\section{Concurrent chemo-radiotherapy}

Chemotherapy consisted of $60 \mathrm{mg} / \mathrm{m} 2$ of paclitaxel given over 1 hour intravenously every week. Patients were pretreated with dexametasone $20 \mathrm{mg}$ given orally on the evening prior to and the morning of paclitaxel infusion. Diphenidramine $25 \mathrm{mg}$ and ranitidine $50 \mathrm{mg}$ were given iv prior to paclitaxel infusion. Carboplatin was given after paclitaxel infusion, for 30 to 60 minutes. Carboplatin dosing to the area under the curve of 1 was calculated using Calvert Formula. Antiemetics were administered as the routine practice. Colony stimulating factors were not used.

\section{Dose modification for toxicity}

Toxicity was graded according to the WHO Criteria. Chemotherapy dose modifications were made for grade 3-4 mucositis or mucositis requiring hospitalization for hydratation/pain management or for grade 3-4 hematologic toxicity. If grade 4 mucositis occurred, chemotherapy was withheld and resumed after the mucositis improved to $\leq$ grade 2 . Carboplatin was withheld if a second episode occurred, and both paclitaxel and carboplatin were withheld if a third episode occurred.

For hematologic toxicity, the dose of paclitaxel and carboplatin was reduced to $50 \%$ for granulocyte count of 500 to $1000 / \mu \mathrm{L}$ and/or platelet count of 50,000 to $75,000 / \mu \mathrm{L}$. Both drugs were withheld if the granulocyte and/or platelet levels dropped below $500 / \mu \mathrm{L}$ and $50,000 / \mu \mathrm{L}$, respectively. Colony stimulating factors were not used. If the weekly granulocyte count was $<500 / \mu \mathrm{L}$, or platelet was $<$ $50,000 / \mu \mathrm{L}$, radiotherapy was withheld until counts, measured twice weekly, recovered to above that level.

\section{Enteral tube feeding was used following physician's prescriptions.}




\section{Radiotherapy}

Radiation treatment was started 3 weeks after the three cycles of Neo Adjuvant Chemotherapy (NACT). Three-dimensional conformal radiotherapy (3D-CRT) was performed on all patients.

Treatment was done using 6 MV photons on a Siemens Primus Linear Accelerator with a multileaf collimator.

For head immobilization in the supine position, a thermoplastic facial mask (MED-TEC) was used. CT-scans were performed for treatment planning.

The clinical target volume (CTV) and organs at risk (OaRs) were outlined on the axial images and were determined according to the CT/RMI findings of the gross tumour and microscopic extension. A 10-mm expansion margin was applied to the CTV to obtain the Planning Target Volume (PTV). The CT images were transferred to the PLATO-Nucletron Planning System, which allowed to obtain Digitally Reconstructed Radiographs (DRR) from the digital image set.

Planned RT consisted of $70 \mathrm{~Gy}$, in 35 fractions, over 7 weeks to all known sites of disease and 50 Gy to sites of potential spread, including the uninvolved neck.

Residual cervical lymphoadenopathy was supplemented with electron beams.

For the first $40 \mathrm{~Gy}$, the nasopharyngeal area and the upper neck were irradiated in one volume with conformal lateral opposing fields. An anterior field was used for the lower neck and supraclavicolar fossa with a laryngeal block.

Thereafter, in 18 cases the radiation beams were rearranged to shrink the field size progressively, after every $10 \mathrm{~Gy}$, to establish gradually a $100 \%$ isodose level that covered the PTV with a total actual dose of $70 \mathrm{~Gy}$.

In 12 patients, in order to reach maximal conformity, after the first 40 Gy of treatment, we employed 5 to 7 fields for the nasopharyngeal region. 
The beam arrangements were determined depending on the anatomical features of the tumour and its relationship with surrounding structures. Wedges were used to improve the conformity of the isodose curves, when necessary.

Maximal doses to the optic pathways, brain stem and spinal cord were limited to $50 \mathrm{~Gy}, 54$ and 45Gy, respectively.

For all patients and treatment plans, dose-volume histograms (DVHs) were calculated for the PTV and OaRs.

Treatment verification was obtained with portal imaging for each new field, and repeated weekly. Treatment interruptions were only allowed for severe normal tissue reactions, such as confluent mucositis.

\section{Statistical methods}

Objective response rate (ORR) represented the primary endpoint and it could be defined as the proportion of patients whose best response was either partial or complete (PR+CR).

Secondary endpoints included disease control rate (DCR), defined as the proportion of patients whose best response was either PR or CR or stable disease (SD), occurrence of grade 3-4 adverse events, as well as progression free survival (PFS) and overall survival (OS).

PFS definition was as follows: the time from the date of the study entry up to the date of first progression, second primary malignancy or death from any causes, whichever came first. Subjects not progressed or died at the time of the analysis were censored at the last disease assessment date. OS was defined as the time from the date of the study entry to the date of death from any cause. Subjects who were not reported as dead at the time of the analysis were censored at the date they were last known to be alive. Survival curves were estimated using the Kaplan-Meier method. All enrolled patients meeting the above described eligibility criteria and not presenting major violations within the duration of our study became subjects of the analysis. Such analysis, indeed, was restricted to patients who received at least one cycle of either induction CHT or CRT. 
Results are expressed as point estimates and their 95\% confidence intervals (95\% CIs). Analysis were carried out using SAS Software, version 9.1 (SAS Institute, Cary, NC). 


\section{Results}

From 2002 to 2007, 62 patients, affected by NPC, have been observed in the participating centres; 30 patients were enrolled in this study protocol. Twenty-two $(73.3 \%)$ patients were males and 8 patients were females (26.7\%) with a median age of 54 years (range 29-69). ECOG performance status was 0 in 27 patients (90\%) and 1 in 3 patients (10\%). WHO histology was as follows: type 2 histology in 3 patients (10\%), type 3 in 27 patients (90\%). Positive tissue EBV DNA was observed in 23 patients (76\%). According to the 2010 AJCC staging system 14 patients had stage III (47\%), 13 patients stage IVa (43\%) and 3 patients stage IV b (10\%) disease; $\mathrm{T}_{3-4}$ lesions were 25/30 (83.3\%) while N2/3 lesions were 21/30 (70\%) (tab 1). Local extension outside nasopharyngeal cavity was as follows: bone lysis in 7 patients (23.3\%), paranasal sinuses involvement in 3 patients (10\%), infratemporal fossa extension in 6 patients (20\%) and intracranial extension in 9 cases $(30 \%)$.

\section{Activity}

All 30 patients completed the planned treatment without protocol violations, and therefore they all were considered assessable for response. After two cycles of CHT no clinical progression was registered and all patients completed the third cycle. After three cycles of neoadjuvant CHT, we registered the following results: 10 patients (33\%) achieved clinical and imaging complete response (CR); eighteen patients (60\%) had a partial response (PR); 6 patients (20\%) achieved PR on the tumour site (T) and no change in regional lymph nodes (N), twelve patients (40\%) achieved a PR on $\mathrm{T}$ and $\mathrm{N}$; two patients (6.6\%) had no change neither in $\mathrm{T}$ nor in $\mathrm{N}$ site. Objective response rate was $93 \%$ (95\% CI $78.4-98.8 \%$ ), while disease control rate was 100\% (95\% CI 94.1\% - 100\%). At the end of CRT, 26 patients (87\%) achieved a clinical CR at both T and regional node and 4 patients (13\%) were in PR (2 patients on T and N, 1 patient on $\mathrm{T}$ only and 1 patient on $\mathrm{N}$ only) for 
an overall response rate of $100 \%$ patients. Objective response rate was 100\% (95\% CI 89.1\%$100 \%)$.

Follow-up at 12 weeks confirmed that 26 patients (87\%) had reached a CR and that 4 patients (13 \%) were in PR according to imaging techniques. One patient with partial response in the neck was submitted to bilateral neck dissection achieving a complete remission.

At a median follow-up time of 49.5 months (range 16-89 months), 6 (20\%) patients progressed and $4(13.3 \%)$ died for tumour. The 3 and 5 year PFS was 80\% (95\% CI 69\% - 99\%) and $75.0 \%$ (95\% CI 55\%-85\%) respectively and the 3 and 5 year OS was $85 . \%$ (95\% CI 69\%-92\%) and 80.0\% (95\% CI 66\%-91\%) respectively (fig. 1). There was a non significant PFS and OS difference between stage III and IV lesions ( $\mathrm{p}>0.05)$.

Five year loco-regional control was $90.3 \%$ (95\% CI 69\%-99\%) and five year distant metastases free survival was $85 \%(95 \%$ CI $72 \%-97 \%)($ Fig 2$)$.

In the follow-up a progression was found in 6 patients and, in particular, two patients had T relapse, one had $\mathrm{T}$ and $\mathrm{N}$ recurrence, three developed distant metastases. All patients ( 3 cases; median time to metastases $=30$ months $;$ range 18-36) with distant metastases (lung 2 , lung + bone 1 ) died after a median period of 27 months (range 21-39 months); they were treated with a median of 2 lines of chemotherapy (range 1-4). T relapses (median time to relapse= 19 months) were treated with reirradiation plus chemotherapy achieving a persistent complete remission. One patient with $\mathrm{T}$ and $\mathrm{N}$ relapse was treated with chemotherapy achieving a PR. The second-line chemotherapy was cisplatin plus epirubicin in all cases.

\section{Toxicity}

A list of acute toxicities with reference to WHO criteria is reported in table 2 and 3. The most clinical relevant side effect of NACT was hematologic toxicity; grade 3-4 neutropenia was observed in 25 cases (83\%) with grade 4 in 15 patients (50.\%) without any episode of febrile neutropenia .G-CSF was administered to 6 patients (20\%). Grade 3 thrombocytopenia occurred in 4 
patients $(13.3 \%)$ without any hemorrhagic episode while grade 3 anaemia was observed in 3 patients $(10 \%)$.

The G 3 non-hematologic toxicity was represented only by oropharyngeal mucositis in 2 patients (6.6\%). All patient received 3 full-dose cycles of CHT.

The most frequent toxicity during CRT was oropharyngeal mucositis occurring in all cases; 16 patients $(53.3 \%)$ developed G3 toxicity and $5(16.6 \%)$ had G4 toxicity. Five of them (16.6\%) required admission to hospital for intravenous hydration and nasal gastric tube feeding. Grade 3-4 haematological toxicity during CRT was as follows: neutropenia in 19 patients $(63.3 \%$; grade 4 in 5 cases $-16.6 \%)$, thrombocytopenia in $5(16.6 \%)$ and anaemia in $5(16.6 \%)$. No febrile neutropenia or hemorrhagic episode was seen. During CRT renal toxicity and neurotoxicity were mild and only 3 patients (10\%) developed grade 3 neuropathy, which was treated with gabapentin and resolved in three months. In 5 patients $(16.6 \%)$ weight loss was significant and they lost $\geq 10 \%$ of their body weight. No toxic deaths were registered during or immediately after treatment. Twenty-seven patients (90\%) received a dose between 66 and $70 \mathrm{~Gy}$; only 3 patients (10\%) interrupted radiation therapy for more than 5 days.

During radiotherapy 189 out of 210 scheduled cycles (90\%) were administered : 21 patients (70\%) received 7 cycles, 2 patients (6.6\%) 6 cycles, 2 patients $(6.6 \%) 5$ cycles and 5 patients (16.6\%) 4 cycles; the relative dose intensity of taxol and carboplatin were $80.1 \%$ and $74.4 \%$, respectively. We registered mild late side effects and sequelae due to CRT according to RTOG criteria. Neck fibrosis was present in 15 patients (50\%) after 6 months of follow-up but it did not take to a relevant clinical problem. RTOG grade 2 xerostomy and disgeusia persisted in 6 patients (20\%) during the first year of follow-up and in 3 patients (10\%) thereafter. Transient swallowing disorders were reported in $3(10 \%)$ patients. We observed sensorineural hearing loss in $2(6.6 \%)$ patients from the beginning of CRT. Such side effect improved slowly after one year from the end of CRT in 1 patient, while it was irreversible in one patient. 


\section{Discussion}

In NPC despite the advances made in clinical therapies of curative RT, with or without concurrent CHT, the 5-year overall survival rates of $\sim 75 \%$ underscore opportunities for improvement, in particular for patients with advanced disease when survival rates decline to $<60 \%$ [14]. The results of the last meta-analysis [15] confirm the role of concurrent chemoradiotherapy as a standard treatment for locoregionally advanced NPC.

A significant benefit was found for overall survival (6\% at 5 years) and event-free survival (10\% at 5 years) with the addition of chemotherapy. The benefit on survival was essentially observed when chemotherapy was administered concomitantly with radiotherapy.

Induction chemotherapy delivered before radiotherapy is an attractive strategy in NPC patients. However, phase III trials, comparing induction chemotherapy with radiotherapy alone [2-5], failed to show an improvement in overall survival, despite a significant reduction in local and distant failures.

In a report of pooled data of two prospective randomized trials [16], the use of induction CHT resulted in a $5.4 \%$ improvement in disease specific survival rate $(\mathrm{p}=0.029)$ at 5 years, while no significant difference was found in the overall survival rates.

Two randomized studies on the use of NACT showed positive results. The VUMCA I study [2] showed significant reduction of both local and distant failures after chemotherapy. Ma et al. [4] also demonstrated significant improvement in local control after NACT.

Hareyama noted that the use of NACT did not result in a significant improvement in disease-free or overall survival, but there was a positive tendency in favour of the NACT for distant metastasis free survival [5]. The negative study by Chan et al. [17] is limited by its low power, since it included only 77 patients. Although the overall results of the Asian-Oceanian Clinical Oncology Association (AOCOA) study is negative [3], an unplanned subgroup analysis showed significant improvement in local control among 53 patients with very large nodes. 
Five recently published studies [6-9] have shown that induction CHT followed by CRT obtained high responses in patients coming from both endemic and non endemic areas: in two trials the authors proposed a three-drugs induction scheme, while in the others cisplatin + epirubicin or paclitaxel and carboplatin were respectively administered.

In four out of five trials the drug administered during radiotherapy was cisplatin alone; only in Oh paper [6] a two drug combination of 5-fluorouracil and hydroxyurea is administered with once-daily radiotherapy on a week-on week-off schedule.

Our clinical post-CRT CR rate $(87 \%)$ is good even if it is probably overestimated unless the radiologists examined serial CT images or the ENT performed multiple biopsies from the tumour area. Our PFS and OS results are superimpose able to the most favourable of these four papers [7$8, \underline{18}]$ conforming the high activity of NACT + CRT even in a non-endemic population.

Our neoadjuvant scheme has achieved a percentage of CR (33\%) similar to the one reported by our group with a cisplatin+epirubicin combination [9] in a superimpose able group of patients.

Probably the two drug administration during RT has given more myelotoxicity and mucositis even if a direct comparison cannot be done. We chose carboplatin instead of cisplatin with the aim of reducing acute toxicity. In a recent paper weekly carboplatin gave less anaemia, renal toxicity, mucositis, weight loss in comparison to 3-weekly cisplatin administration.[19]

Severe nausea/vomiting was less frequent in carboplatin+taxol group ( $0 \%$ vs $12.5 \%)$. Late toxicity was superimpose able in our two series.

Our percentage of distant metastases is quite low (10\%) even if it is possible to suppose that an induction CHT with three-drugs scheme or a molecular targeted therapy integration may improve these results, as reported in other head and neck tumours, different from nasopharyngeal region; however such observation needs to be evidenced by mean of future randomized trials. According to our previously reported experience in a very similar series [9] we can draw the following conclusions: 
1) NACT with carboplatin and taxol is very well tolerated with a clinical impact superimpose able to cisplatin + epirubicin;

2) A two drug combination during conventional radiotherapy is a feasible treatment with a good compliance, an high relative dose intensity and no significant life-threatening toxicity;

3) A two drug combination during radiotherapy has an higher acute severe toxicity (neutropenia, anemia, oropharyngeal mucositis, weight loss) compared to cisplatin every three weeks ;

4) Tumour recurrence can be effectively treated with re-irradiation $+\mathrm{CHT}$, obtaining long lasting complete remissions: this is in accordance with recent reports [20-22]. Although the small series hereby presented, NACT with carboplatin + taxol followed by concomitant weely carboplatin + taxol + conventional radiotherapy seems to be a safe and effective treatment for a non endemic population affected by locoregional advanced NPC; however this approach needs further phase 3 trials. 


\section{REFERENCES}

1. Guigay J, Temam S, Bourhis J, et al. (2006) Nasopharyngeal carcinoma and therapeutic management: the place of chemotherapy. Ann Oncol 17 (suppl 10):x304-x307.

2. International Nasopharynx Cancer Study Group. VUMCA I trial: preliminary results of a randomized trial comparing neoadjuvant chemotherapy (cisplatin, epirubicin, belomycin) plus radiotherapy vs. radiotherapy alone in stage IV (N2, M0) undifferentiated nasopharyngeal carcinoma: a positive effect on progression-free survival. Int J Radiat Oncol Biol Phys $1996 ; 35: 463-9$

3. Chua DT, Sham JS, Choy D, et al. (1998) Preliminary report of the Asian-Oceanian Clinical Oncology Association randomized trial comparing cisplatin and epirubicin followed by radiotherapy versus radiotherapy alone in the treatment of patients with locoregionally advanced nasopharyngeal arcinoma. Cancer 83:2270-2283.

4. Ma J, Mai HQ, Hong MH, et al. (2001) Results of a prospective randomized trial comparing neoadjuvant chemotherapy plus rsdiotherapy with radiotherapy alone in patients with locoregionally advanced nasopharyngeal carcinoma. J Clin Oncol 19:1350-1357

5. Haeryama M, Sakata K, Shirato H, et al. (2002) A prospective randomized trial comparing neoadjuvant chemotherapy with radiotherapy alone in advanced nasopharyngeal carcinoma. Cancer $94: 2217-2223$ 
6. Oh JL, Vokes EE, Kies MS, et al. (2003) Induction chemotherapy followed by concomitant chemoradiotherapy in the treatment of locoregionally advanced nasopharyngeal cancer. Ann Oncol 14:564-569.

7. Rischin D, Corry J, Smith J, et al. (2002) Excellent disease control and survival in patients with advanced nasopharyngeal cancer treated with chemoradiation. J Clin Oncol 20:1845-52

8. Al-Amro A, Al Rajhi N, Khafaga J, et al. (2005) Neoadiuvant chemotherapy followed by concurrent chemoradiation therapy in locally advanced nasopharyngeal carcinoma. Int J Radiat Oncol Biol Phys 62(2):508-513

9. Airoldi M, Gabriele AM, Garzaro M, et al. (2009) Induction Chemotherapy with Cisplatin and Epirubicin followed by radiotherapy and concurrent Cisplatin in locally advanced nasopharyngeal carcinoma observed in a non-endemic population. Radiother Oncol 92(1):105-10

$\underline{\text { 10. Airoldi M, Pedani F, Marchionatti S et al. (2002) Carboplatin plus taxol is an effective third-line }}$ $\underline{\text { regimen in recurrent undifferentiated nasopharyngeal carcinoma. Tumori 88(4):273-6 }}$

11.Tan EH, Khoo KS, Wee J et al. (1999) Phase II trial of a paclitaxel and carboplatin combination in Asian patients with metastatic nasopharyngeal carcinoma. Ann Oncol 10(2):235-7

$\underline{\text { 12. Yeo W, Leung TW, Chan AT et al. (1998) A phase II study combination paclitaxel and }}$ carboplatin in advanced nasopharyngeal carcinoma. Eur J Cancer 34(13):2027-31

13. Seidman AD. (1998) One-hour paclitaxel via weekly infusion: dose-density with enhanced therapeutic index. Oncology 12:19-22 
14. Lee AW, Sze WM, Au JS, et al. (2005) Treatment results for nasopharyngeal carcinoma in the modern era : the Hong Kong experience. Int J Radiat Oncol Biol Phys 61:1107-1116.

15. Baujat B, Audry H, Bourhis J, et al. (2006) Chemotherapy in locally advanced nasopharyngeal carcinoma: an individual patient data meta-analysis of eight randomized clinical trials and 1753 patients. Int J Radiation Oncology Biol Phys 64:47-56

16. Chua DT, Ma J, Sham JS, et al. (2005) Long-term survival after cisplatin-based induction chemotherapy and radiotherapy for nasopharyngeal carcinoma : a pooled data analysis of two phase III trials. J Clin Oncol 23:1118-24

17. Chan AT, Teo PM, Leung TW, et al. (1995) A prospective randomized study of chemotherapy adjunctive to definitive radiotherapy in advanced nasopharyngeal carcinoma. Int J Radiat Oncol Biol Phys 33:569-77

18. Chan ATC, Ma BBY, Lo D, et al. (2004) Phase II study of neoadjuvant carboplatin and paclitaxel followed by radiotherapy and concurrent cisplatin in patients with locoregionally advanced nasopharyngeal carcinoma: therapeutic monitoring with plasma Epstein-Barr Virus DNA. J Clin Oncol 22;15:3053-3060

19. Chitapanarux I, Lorvidhaya W, Kamnerdsupaphon P, et al. (2007) Chemoradiation comparing cisplatin versus carboplatin in locally advanced nasopharyngeal cancer: randomised, non inferiority, open trial. Eur J Cancer 43(9):1399-406

20. Kramer NM, Horwitz EM, Cheng J, et al. (2005) Toxicity and outcome analysis of patients with recurrent head and neck cancer treated with hyperfractionated split-course reirradiation and 
concurrent cisplatin and paclitaxel chemotherapy from two prospective phase I and II studies. Head Neck 27(5):406-14

21. Spencer SA, Harris J, Weeler RH, et al. (2008) Final report of RTOG 9610, a multi-institutional trial of reirradiation and chemotherapy for unresectable recurrent squamous cell carcinoma of the head and neck. Head Neck 30(3):281-8

22. Langer CJ, Harris J, Horwitz EM, et al. (2007) Phase II study of low dose paclitaxel and cisplatin in combination with split course concomitant twice-daily reirradiation in recurrent squamous cell carcinoma of the head and neck: results of Radiation Therapy Oncology Group Protocol 9911. J Clin Oncol 25(30):4800-5 
Table 1 - Tumour stage versus Node stage (AJCC Staging 2010)

\begin{tabular}{l|cccc|c}
\hline & $\mathrm{T} 1$ & $\mathrm{~T} 2$ & $\mathrm{~T} 3$ & $\mathrm{~T} 4$ & Total \\
\hline $\mathrm{N} 0$ & -- & -- & 1 & 8 & 9 \\
$\mathrm{~N} 1$ & -- & -- & 0 & 0 & 0 \\
$\mathrm{~N} 2$ & $\underline{3}$ & $\underline{1}$ & 9 & 5 & 18 \\
$\mathrm{~N} 3^{*}$ & 1 & -- & -- & 2 & 3 \\
\hline Total & 3 & 2 & 10 & 15 & 30 \\
\hline
\end{tabular}

*All patients were N3a 
Table 2 - Acute toxicity (WHO criteria), maximum toxicity per patient during induction CHT

\begin{tabular}{|c|c|c|c|c|}
\hline WHO Grade & 0 & $1-2$ & 3 & 4 \\
\hline Neutropenia & $0(0 \%)$ & $5(16.7 \%)$ & $10(33.3 \%)$ & $15(50 \%)$ \\
\hline Thrombocytopenia & $2(6.6 \%)$ & $24(80.0 \%)$ & $4 \quad(13.3 \%)$ & $0(0 \%)$ \\
\hline Anemia & $2(6.6 \%)$ & $15(50.0 \%)$ & $3(10.0 \%)$ & $0(0 \%)$ \\
\hline Nausea/Vomiting & $15(50.0 \%)$ & $15(50.0 \%)$ & $0 \quad(0 \%)$ & $0(0 \%)$ \\
\hline Anorexia & $23(76.7 \%)$ & $7(23.3 \%)$ & $0(0 \%)$ & $0(0 \%)$ \\
\hline Oropharyngeal Mucositis & $21(70.0 \%)$ & $7(23.3 \%)$ & $2(6.6 \%)$ & $0(0 \%)$ \\
\hline Alopecia & $6(20 \%)$ & $24(80.0 \%)$ & & \\
\hline Skin & $0(0 \%)$ & -- & -- & -- \\
\hline Cardiotoxicity & $0(0 \%)$ & -- & -- & -- \\
\hline Renal toxicity & $0(0 \%)$ & -- & -- & -- \\
\hline Neurotoxicity & $0(0 \%)$ & -- & -- & -- \\
\hline Weight loss & $36(87.7 \%)$ & $4(13.3 \%)$ & -- & -- \\
\hline
\end{tabular}

*Febrile neutropenia in no patient . 
Table 3 - Acute toxicity (WHO criteria), maximum toxicity per patient, during CRT

\begin{tabular}{|c|c|c|c|c|}
\hline WHO Grade & 0 & $1-2$ & 3 & 4 \\
\hline Neutropenia & $0(.0 \%)$ & $11(36.6 \%)$ & $16(53.3 \%)$ & $3(10 \%)$ \\
\hline Thrombocytopenia & $10(33.3 \%)$ & $17(56.6 \%)$ & $3(10 \%)$ & $0(0 \%)$ \\
\hline Anaemia & $8(26.6 \%)$ & $17(56.6 \%)$ & $5(16.6 \%)$ & $0(0 \%)$ \\
\hline Nausea/Vomiting & $18(60.0 \%)$ & $12(40.0 \%)$ & $0(0 \%)$ & $0(0 \%)$ \\
\hline Oropharyngeal Mucositis & $0(0 \%)$ & $9(30 \%)$ & $16(53.3 \%)$ & $5(16.6 \%)$ \\
\hline Alopecia & $6(20 \%)$ & $24(80 \%)$ & & \\
\hline Skin & $2(6.6 \%)$ & $21(70.0 \%)$ & $7(23.3 \%)$ & $0(0 \%)$ \\
\hline Cardiotoxicity & $30(100 \%)$ & -- & -- & \\
\hline Renal toxicity & $28(93.3 \%)$ & $2(6.6 \%)$ & -- & \\
\hline Neurotoxicity & $12(40 \%)$ & $15(50 \%)$ & $3(10 \%)$ & $0(0 \%)$ \\
\hline Weight loss & $32(80.0 \%)$ & $8(20.0 \%)$ & $5(16.6 \%)$ & $0(0 \%)$ \\
\hline NGT & \multicolumn{4}{|c|}{ yes 5 pts $(16.6 \%)$} \\
\hline
\end{tabular}




\section{Figure Legends:}

Fig. 1 Overall Survival (OS) and Disease Free Survival (PFS) - 30 patients with a median followup of 49.5 months.

Fig. 2 Local Recurrence Disease Free Survival (LRDFS) and Distant Metastases Disease Free Survival (DMDFS) - 30 patients with a median follow-up of 49.5 months.
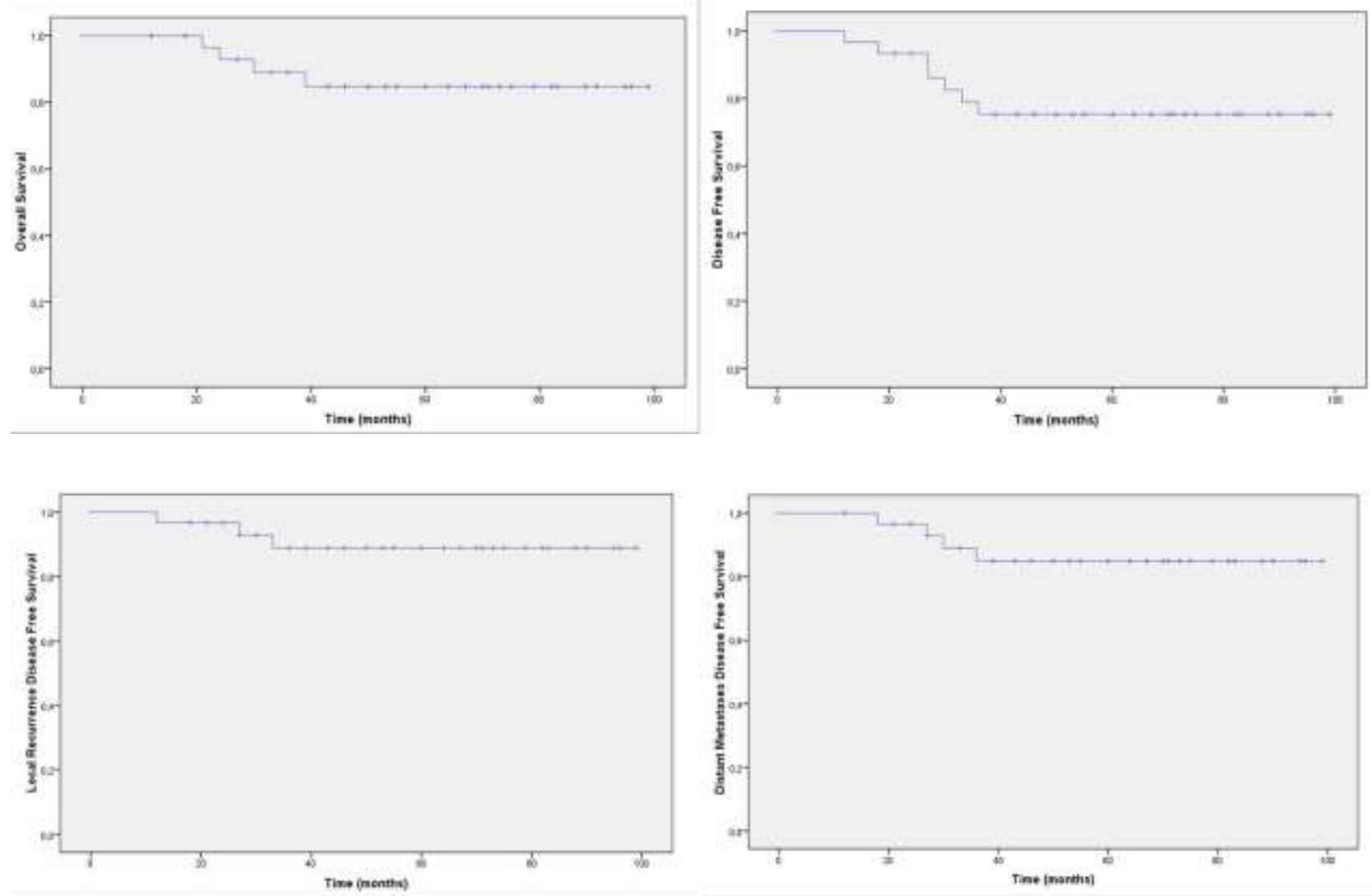